\title{
FIRST: Fast Iterative Reconstruction Software for (PET) tomography
}

\author{
J L Herraiz ${ }^{1}$, S España ${ }^{1}$, J J Vaquero ${ }^{2}$, M Desco ${ }^{2}$ and J M Udías ${ }^{1}$ \\ ${ }^{1}$ Dpto. Física Atómica, Molecular y Nuclear, Universidad Complutense de Madrid, Spain \\ ${ }^{2}$ Unidad de Medicina y Cirugía Experimental, Hospital GU Gregorio Marañón, Madrid, Spain \\ E-mail: jose@nuc2.fis.ucm.es
}

\begin{abstract}
Small animal PET scanners require high spatial resolution and good sensitivity. To reconstruct high-resolution images in 3D-PET, iterative methods, such as OSEM, are superior to analytical reconstruction algorithms, although their high computational cost is still a serious drawback. The higher performance of modern computers could make iterative image reconstruction fast enough to be viable, provided we are able to deal with the large number of probability coefficients for the system response matrix in high-resolution PET scanners, which is a difficult task that prevents the algorithms from reaching peak computing performance. Considering all possible axial and in-plane symmetries, as well as certain quasi-symmetries, we have been able to reduce the memory requirements to store the system response matrix (SRM) well below $1 \mathrm{~GB}$, which allows us to keep the whole response matrix of the system inside RAM of ordinary industry-standard computers, so that the reconstruction algorithm can achieve near peak performance. The elements of the SRM are stored as cubic spline profiles and matched to voxel size during reconstruction. In this way, the advantages of 'on-the-fly' calculation and of fully stored SRM are combined. The on-the-fly part of the calculation (matching the profile functions to voxel size) of the SRM accounts for 10-30\% of the reconstruction time, depending on the number of voxels chosen. We tested our approach with real data from a commercial small animal PET scanner. The results (image quality and reconstruction time) show that the proposed technique is a feasible solution.
\end{abstract}

\section{Introduction}

There is a strong demand for fast and accurate reconstruction procedures for high-resolution, high-sensitivity PET scanners. These systems, typically used in small animal PET studies, 
are designed with the goal of optimizing spatial resolution while maintaining good detection sensitivity. They consist of several pairs of opposite scintillation detectors, each of which is coupled to an array of small crystals arranged in a static ring or in a rotating device. The detector ring diameter and the size of the field of view (FOV) are usually less than $20 \mathrm{~cm}$. Since spatial resolutions in the range of $1 \mathrm{~mm}$ are required, these detectors employ many small crystals, thus leading to a very large number of lines of response (LORs), defined by every possible pair of crystals. Moreover, 3D acquisitions (and reconstructions) are mandatory due to sensitivity requirements.

Statistical 3D reconstruction methods such as expectation maximization (EM) (Shepp and Vardi 1982, Browne and De Pierro 1996) have shown superior image quality than conventional analytic reconstruction techniques. Moreover, EM has some desirable properties such as conservation of the number of counts, non-negativity, good linearity and dynamic range. One of the key advantages of statistical reconstructions is the ability to incorporate accurate models of the PET acquisition process through the use of the system response matrix (SRM). However, SRM for 3D systems are of the order of several billions of elements, which imposes serious demands for statistical iterative methods in terms of the time required to complete the reconstruction procedure and the computer memory needed for the storage of the SRM. We have designed, developed, implemented and tested a new EM-based reconstruction methodology that provides fast reconstructions while remaining very flexible. With our approach, the usual difficulties of iterative reconstruction methods regarding the large size of the SRM or the use of unrealistic estimates of it are overcome by means of a compressed and realistic SRM. The efficiency of the proposed method relies on the design and method of storing this SRM. The imaging process of obtaining the $y(i)$ counts on each of the $i$ pair of detectors, from an object discretized in $x(j)$ voxels, can be described by the operation $y(i)=\sum_{j} A(i, j) x(j)$, where $A(i, j)$ is the SRM, the vector $x(j)$ corresponds to the voxelized image and $y(i)$ to the measured data. Each element $A(i, j)$ is defined as the probability of detecting an annihilation event coming from image voxel $j$ by a detector pair $i$. This probability depends on factors such as the solid angle subtended by the voxel to the detector element, the attenuation and scatter in the source volume and the detector response characteristics.

The forward projection operation just introduced above estimates the projection data from a given activity distribution of the source. Backward projection is the transposed operation of forward projection; it estimates a source volume distribution of activity from the projection data. The operation corresponds to $b(j)=\sum_{i} A(i, j) y(i)$, where $b(j)$ denotes an element of the backward projection image. Both the forward and backward projection operations require the knowledge of the SRM (Frese et al 2003, Rafecas et al 2004). Iterative reconstruction algorithms repeatedly use the forward and backward projection operations, which are the most time-consuming parts of iterative reconstruction programs. Some implementations trade accuracy for speed by making approximations that neglect some physical processes, such as positron range, scatter and fractional energy collection at the scintillators or visible light loses in the detectors (Vaquero et al 2004, Lee et al 2000, Yamaga and Murayama 2002-2003). This approach simplifies these operations to increase speed, but this trade-off often leads to non-optimal images.

The evaluation and storage of SRM elements is a very active subject of research. Ideally, the SRM could be calculated, using MC methods (Rafecas et al 2004) or from empirical data (Frese et al 2003), and stored once and for all before the beginning of the reconstruction process. In practice, memory requirements for doing this have become prohibitive so far. A number of methods have been proposed to compute and handle huge sparse matrices like the SRM. Some implementations compute the elements $A(i, j)$ on the fly, only if and when they are required (Kudrolli et al 2002), thus avoiding the need to store the whole SRM. 
Table 1. Vista parameters.

\begin{tabular}{ll}
\hline Ring diameter & $11.8 \mathrm{~cm}$ \\
Aperture & $8 \mathrm{~cm}$ \\
Axial FOV & $4.8 \mathrm{~cm}$ \\
Number of DOI detector modules & 36 position-sensitive PMTs \\
Number of dual-scintillator DOI elements & 6084 \\
Crystal array pitch & $1.55 \mathrm{~mm}$ \\
Total number of crystals & 12168 \\
Total number of 3D coincidence lines & $28.8 \times 10^{6}$ \\
\hline
\end{tabular}

In other approaches, the SRM has been factorized as a product of independent contributions: geometry, attenuation and detector sensitivity (Qi et al 1998). The simplifications required by on-the-fly or factorized calculations often overlook important effects (Lee et al 2004).

Due to the fact that the SRM is very large but sparse, it may be kept on disk by using sophisticated storage schemes and taking advantage of system symmetries (Johnson and Sofer $1999 b)$ to reduce the size of the SRM to a few tenths of gigabytes. Due to the fact that accessing the SRM from disk for every forward and backward projection operation is very slow, this considerably slows down the reconstruction. Our approach involves compressing the SRM to the extent that enables its allocation in the RAM memory of industry-standard computers, avoiding disk access during reconstruction. In this way, it is possible to achieve a sustained performance of around $50 \%$ of the theoretical peak computing capability of the processors.

\section{System response matrix $(\mathrm{SRM})$}

The SRM is composed of all the $V \times L$ probability elements $A(i, j), i=1, \ldots, L, j=$ $1, \ldots, V$, representing the probability of detecting an event coming from voxel $V(j)$ at detector LOR (line of response) $L(i)$. Forward and backward projections require the knowledge of all of these elements. This matrix depends on factors such as the physics of beta decay, attenuation and scatter in the source volume, solid angle subtended from voxel to detector element and intrinsic detector response characteristics. For a reconstruction method to be accurate, all these effects should be considered. The equipment used in this study is an eXplore Vista-DR (GE) small animal PET scanner (Vaquero et al 2004). It is a ring-type scanner with a diameter of $11.8 \mathrm{~cm}$, a transverse field of view (FOV) of $6.8 \mathrm{~cm}$ and an axial FOV of $4.6 \mathrm{~cm}$. Vista technology is based on scintillator detector modules with depth-of-interaction (DOI) capabilities (Lee et al 2000) arranged in single (SR) or double rings (DR). The detector modules are composed of a $13 \times 13$ crystal array with $1.55 \mathrm{~mm}$ pitch size. The number of LORs in this scanner is over $2.8 \times 10^{7}$ (see table 1 ). DOI determination enables spatial resolution and sensitivity to be improved simultaneously (Yamaga and Murayama 2002-2003).

From the data of table 1 and at nominal image resolution of $175 \times 175 \times 62$ voxels (near 1.9 millions of voxels), the number of elements in the SRM (number of LORs $\times$ number of voxels) is of the order of $5 \times 10^{13}$. Storing all the elements of the SRM would require more than 10 TB (Rafecas et al 2004). This exceeds the resources of any ordinary workstation, making it necessary to disregard all redundant elements and to perform approximations in order to be able to store the SRM in the limited amount of RAM of ordinary workstations. Three techniques have been used to achieve this goal: null or almost-null element removal (matrix sparseness), intensive use of system symmetries and compression of the resulting SRM employing quasi-symmetries. 


\subsection{Matrix sparseness}

Every detector pair can receive coincidence counts only from a relatively small portion of the FOV. Thus, most matrix elements of the SRM are null and only the non-zero elements should be stored, reducing considerably the storage requirements. To estimate how many non-zero elements of the SRM have to be taken into account, we proceed as follows: the voxels connected to a given LOR (that is, the voxels from which positron decay can produce with non-negligible probability a valid coincidence count in the detectors that define the LOR) constitute the so-called channel of response or CHOR (Michel et al 2000) for that LOR. Extensive simulations determine the maximum size of the CHOR needed and only the SRM elements that are part of some CHOR are stored. We consider a voxel not connected to a LOR (i.e., not being part of the CHOR) if the probability that a positron emitted from that voxel yields a count in the corresponding detector pair is smaller than one-thousandth of the maximum value of all the voxels for such given CHOR. For the scanner considered here we use a nominal number of voxels of $175 \times 175 \times 62$ in $X Y Z$ to cover the FOV. This yields a voxel size of $0.38 \times 0.38 \times 0.76 \mathrm{~mm}^{3}$ (see table 1) and an average number of voxels in a CHOR of about 6000 for a typical CHOR size of 150 (longitudinal) $\times 10$ (transverse width of the CHOR in the transaxial $X Y$ plane of the scanner) $\times 4$ (transverse width in the axial or $Z$ direction scanner) voxels (Johnson and Sofer 1999b). With these choices, the number of non-zero elements of the SRM is then $28.8 \times 10^{6}$ LORs $\times 6000$ connected voxels on average or $10^{11}$ elements. That is, only around $0.2 \%$ of the elements of the SRM are non-zero. Yet, storing these non-zero elements as floats ( 4 bytes per SRM element) will require about $600 \mathrm{~GB}$ of disk space, still too high for the current RAM amount of industry-standard computers.

\subsection{System symmetries}

An additional reduction factor of approximately 40 in the number of non-null SRM elements that needed to be stored can be achieved by assuming that (exact) axial (translation and reflection) and in-plane symmetries exist (Johnson and Sofer 1999b). Voxels were chosen in an orthogonal grid oriented along $X, Y$ and $Z$-axis, with $Z$ being the axis of the scanner. If an integer number of voxels is employed for the width of the CHOR in the $Z$-axis, then there is a $Z$-translation symmetry, due to the fact that voxels in the same relative position of the CHOR and belonging to parallel CHORs should have equal values (see figure 1).

We must however note that although our SRM exhibits indeed this translational symmetry, in real scanners, due to edge and block effects, it is only an approximate symmetry.

There is also another axial symmetry, Z-reflection symmetry. Using both parallel and reflection $Z$-symmetries, the number of elements to be stored is reduced considerably. Each pair of blocks has $(2 \times 13 \times 13) \times(2 \times 13 \times 13)$ LORs, but using symmetries, only $2 \times 13 \times(2 \times 13 \times 13)$ need to be stored. A factor 13 of reduction in the space needed is achieved.

Another symmetry, reflection symmetry among blocks in the $X Y$ plane, also holds. Using this, the number of pairs of detectors that have CHORs with different values is reduced by a factor of 3. Using them all, as we have already mentioned, these symmetries allow to reduce by a factor or near 40 the number of different elements of the SRM that must be stored. Storage needs can thus be reduced to a few (slightly less than 10, for the scanner we consider here) $\mathrm{GB}$, small enough to fit in hard disks, yet too much to be maintained in RAM.

\subsection{Compressed SRM}

The last step of the method we propose here uses additional non-exact symmetries, or quasisymmetries, in order to obtain additional reduction of the SRM. If we allow for relatively 


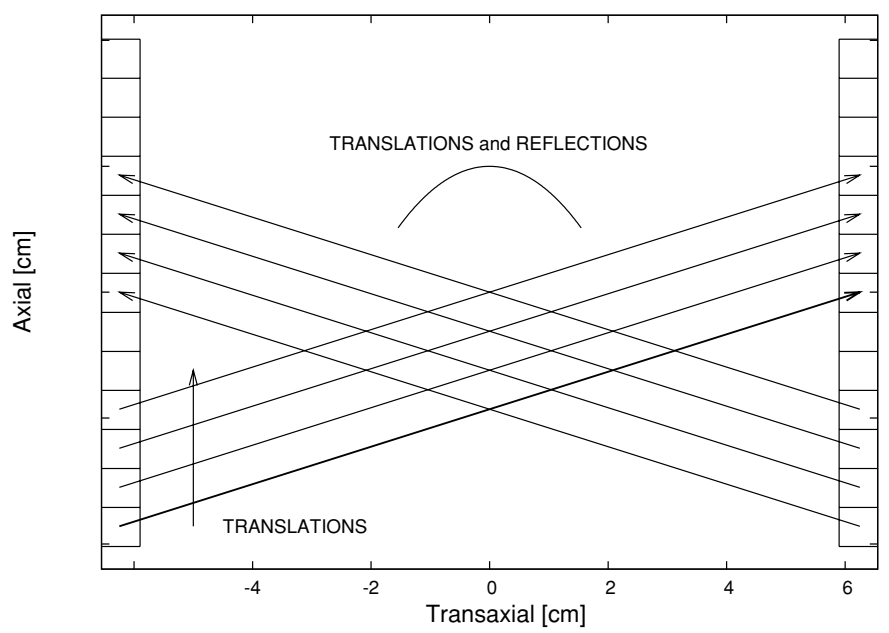

Figure 1. Schematic drawing of a Vista small animal PET scanner detector pair, showing the (exact) translation and reflection symmetries employed in this work. All the elements of the SRM belonging to parallel LORs are, by symmetry, equivalent.

small differences between quasi-symmetric elements of the SRM (versus no difference a priori in the case of the exact symmetries), we can group certain LORs into sets of the same quasi-symmetry class. The differences between the elements of the SRM for LORs belonging to a given class should be much smaller than between LORs from different classes. Quasisymmetry classes can be obtained, for instance, by grouping together LORs from crystals with different, but close, LOR-crystal orientations. The differences among the elements of the same quasi-symmetry class are about $5-10 \%$, depending on the amount of compression (reduction in size) desired.

In figures 2-4, we illustrate this procedure with an example taken from our simulations. MC events were generated at different positions inside a CHOR. As shown in figure 2, LORs 1-3 are parallel or almost parallel to the crystals and thus the probability values along these three CHORs should be very similar. Analogously, LORs 4-6 have a large LOR-crystal angle, similar for the three of them. In figure 3, longitudinal profiles along these LORs are shown. We can see indeed that the results of the MC simulations for the calculation of the probabilities for LORs 1-3 (and 4-6), shown by the data points (that include statistical error bars), are very similar. We could even say that these are the same within the error bars. In figure 4, now the profiles of the CHORs along the transverse direction to the LORs ( $s$-coordinate) are shown at several values of the $l$-coordinate. As in the case of the longitudinal profiles, we can see that the results of the simulated data for the near equivalent LORs 1-3 or 4-6 are very similar. Also we realize that the variation of probability inside a CHOR is smooth, which allows us to fit the simulated points to profiles with an smooth interpolating cubic spline. The differences among the results of the interpolating curves for LORs 1-3 are marginal and the three interpolating curves could be considered identical within the statistical error bars of the MC points. A similar observation can be made for CHORs 4-6. Our quasi-symmetry assumption means that we will employ the same profile functions for CHORs 1-3 that belong to the same quasisymmetry class. CHORs $4-6$ belong to another quasi-symmetry class and be represented by one set of profile functions, different from those of the other quasi-symmetry classes.

Depending on the geometry of the system, the use of quasi-exact symmetries allows us to obtain a number of quasi-equivalent LOR classes (that is, CHORs with non-equivalent values) which may be 9 (in the example so far discussed) to 25 (for up to five different 


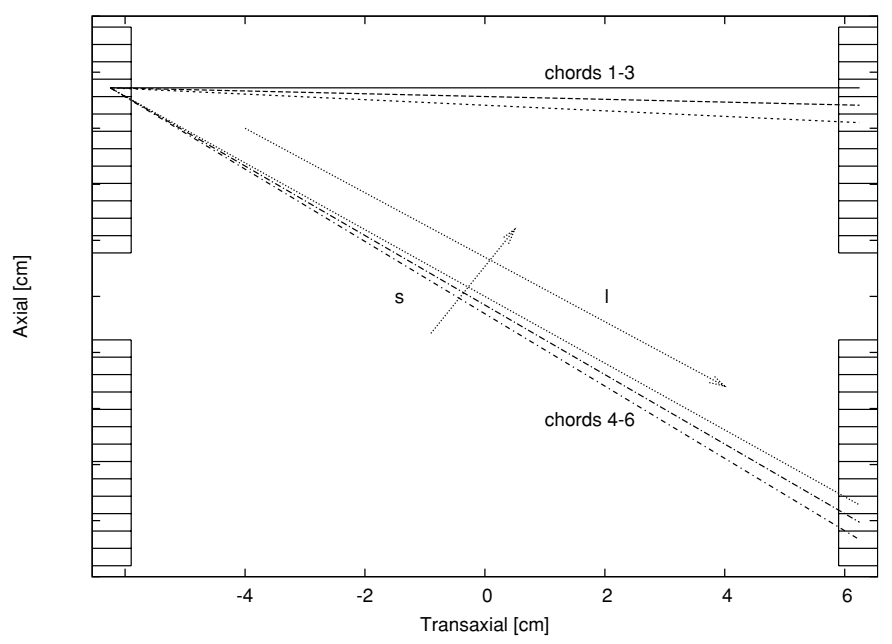

Figure 2. Schematic representation of several lines of response (LOR) considered for the discussion on quasi-symmetries. Three LORs (numbered 1-3 from top to bottom) with a small relative LORcrystal angle and three (numbered 4-6, also from top to bottom) with large relative LOR-crystal angle are depicted. $l$ and $s$ are the coordinates along the LOR direction and normal to it, respectively.

LOR-crystal angles in the same quasi-symmetry class, allowing for larger differences among the profiles within the same quasi-symmetry class) times smaller than the number of classes obtained with only exact symmetries. The elements of this notably reduced SRM are encoded as transverse and longitudinal profile functions obtained by cubic spline interpolation of MC sampled points. For each transversal or longitudinal profile, MC estimates of probability at 20 points along or across the CHORs are employed to determine the cubic spline profile functions. At reconstruction time, the probability element of the SRM corresponding to each voxel inside a CHOR is obtained by interpolation of the profile functions. If the voxel size chosen is large, we average several values interpolated from the profile functions at different points inside the voxel, in order to compute the probability for each voxel. The interpolation and averaging of probability inside each voxel from profile functions is compared with the results of averaging of points obtained directly from the MC simulations. From these comparisons, we conclude that for a number of voxels above or below a factor of 3 of the nominal number of voxels of $175 \times 175 \times 62$ the interpolation procedure differs typically by less than 5\% from the results of direct MC simulation in the example shown in figures 2-4 (three different LOR-crystal angles) and by less than $10 \%$ for larger quasi-symmetry class (five different LOR-crystal angles).

In short, the same quasi-equivalent profiles can be used to build the non-zero SRM elements for a reasonable range of voxel sizes. The optimal voxel size that should be employed for each reconstruction may be different depending for instance on the number of counts of a particular acquisition. This profile function approach makes it possible to generate reconstructions with different voxel sizes without the need to recompute the SRM elements. Eventually, this process leads to a compressed SRM that fits in 30-150 MB, depending on the degree of quasi-symmetry assumed.

To end this section on compressing the SRM, we comment on the storing strategy that is also useful to save space. All the cubic spline coefficients for the profiles of a quasi-symmetry class (or superCHOR) are rescaled in order to convert (and store) them as integer values. Two bytes are employed to represent every coefficient, which allows us to represent ratios of more 


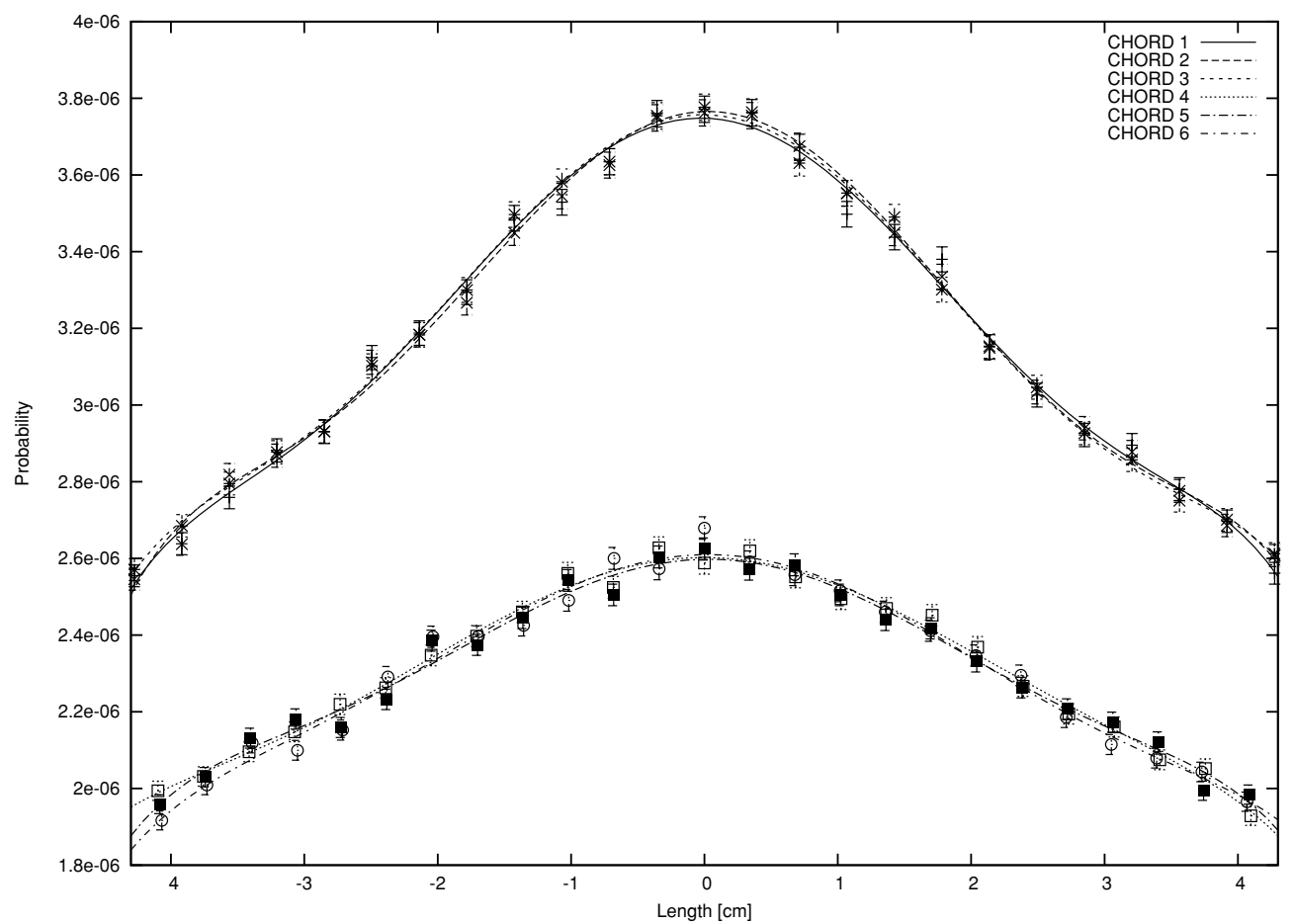

Figure 3. Longitudinal profile of the probability elements for the LORs shown in figure 2. The probability of detection of a coincidence count in LORs 1-6 per every positron decay in the axis of the CHOR as a function of the distance to the centre of the CHOR is shown. The data points represent the results for the MC simulation described in the text, the error bars are given by the statistical uncertainty. Symbols employed are as follows: 1 , plus (+) sign; 2 , times $(\times)$ sign; 3 , star (*) sign; 4, empty square; 5 , solid square; 6 , empty circle. Profiles fitted to the points by means of cubic splines are also shown. The small crystal-LOR angle (1-3) profiles are very similar among them, but rather different from the large crystal-LOR angle ones (4-6).

than 60000 to 1 inside the same CHOR. The scale factors (maximum and minimum values of the coefficients for all the profiles in each superCHOR) are also recorded as two additional floating numbers. During reconstruction, the integer values are converted into the adequate float ones on the fly. The FOV is divided in voxels arranged in an orthogonal grid. For a given CHOR, voxels are visited from bottom to top, left to right and back to front directions. Every voxel in the superCHOR is visited in order and the SRM element corresponding to that voxel-LOR combination is obtained by interpolation of the cubic spline profiles. Then, the superCHOR values are stored as a list of numbers formed by the probabilities of each voxel of the superCHOR visited in the assumed order. Once the superCHOR is obtained (decompressed) on the fly, all the operations (forward or backward projections) that involve the CHORs in the quasi-symmetry class are performed.

\subsection{MC simulation}

Given the fact that the compressed SRM fits in RAM, it does not need to be computed during reconstruction, nor read from disk once loaded in memory at the beginning of the reconstruction. Thus, the SRM can be computed using a very realistic model and stored once and for all. Monte Carlo (MC) methods are, in principle, well suited to provide realistic estimates of SRM elements. In our case, we use our own MC model that includes scatter and 


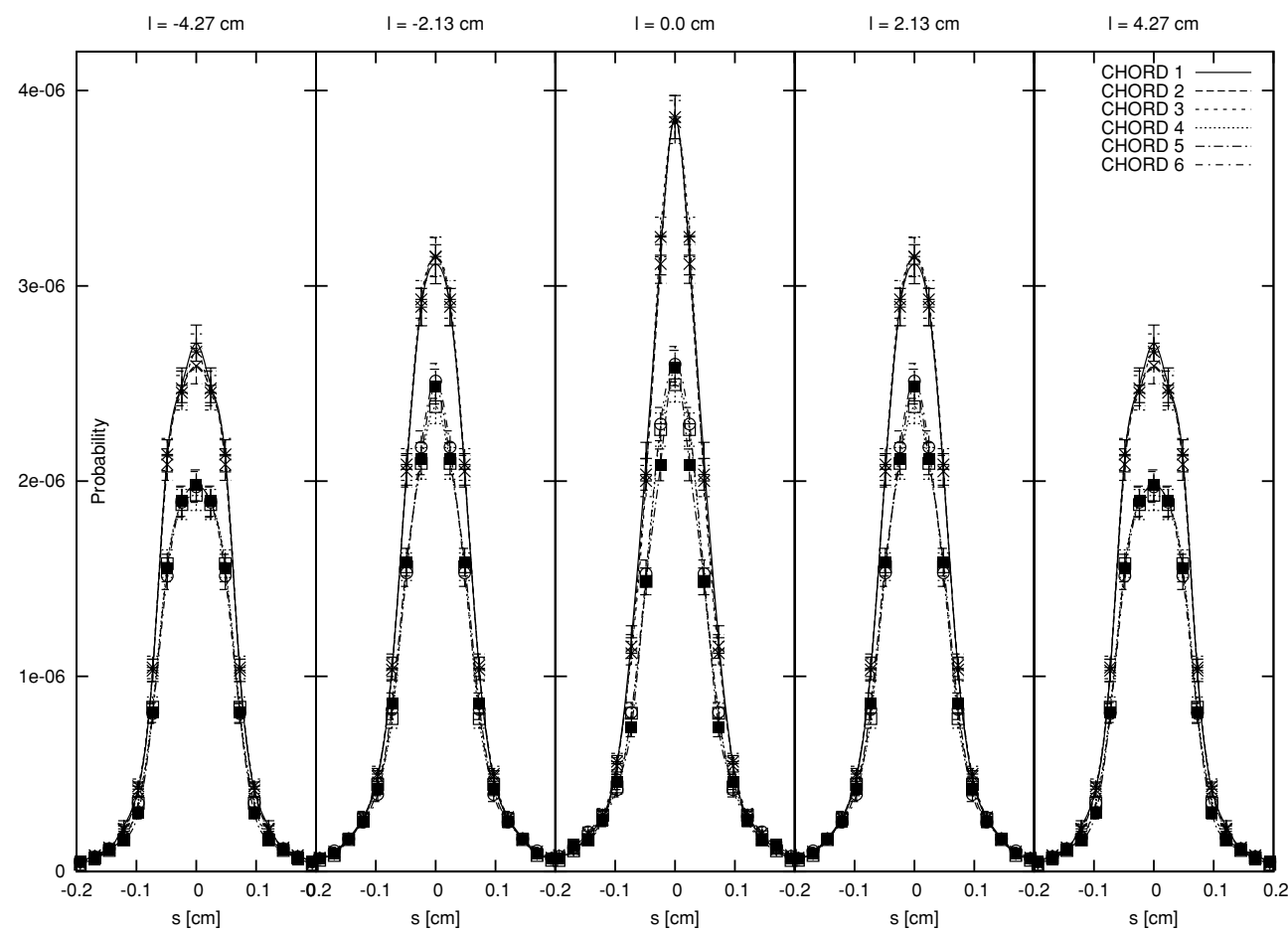

Figure 4. Transverse profiles of LORs 1-6 of figure 2. Data points and curves as in figure 3 . Several profiles at different distances $l$ to the centre of the LOR are shown. The transversal width of the CHORs as shown in the figures is around $4 \mathrm{~mm}$ or three crystal widths.

incomplete collection of energy in the scintillator crystals, positron range and non-collinearity effects. Positron range is dependent on the object. In order to incorporate its effect in the SRM, in our simulations the range is computed assuming that water uniformly fills the whole FOV. Most importantly, we also include the scatter of gamma photons when they reach the scintillator crystals. The comparison of simulated phantoms with actual acquisitions reveals that the simulation is very realistic indeed.

A large number of simulated events are accumulated until the statistical uncertainty is below 5\% at the centre of a typical LOR. Several weeks of computing time were required for the calculation of the SRM in a cluster composed of 12 industry-standard workstations. The total time employed for the full MC simulation is equivalent to 180 days of a single Pentium IV $3.0 \mathrm{GHz}$ workstation.

\section{Iterative image reconstruction algorithms}

To test the accuracy of the compressed SRM we have obtained, we use one of the most widely applied algorithm for finding the maximum-likelihood (ML) estimation of activity $x$ given the projections $y$, that is expectation maximization (EM). This was first applied to the emission tomography problem by Shepp and Vardi (1982). ML, though, is a general statistical method, formulated as a method of solving many different optimization problems.

Usually, iterative algorithms obtained from the ML statistical model assume that the data being reconstructed retain Poisson statistics. However, to preserve the Poisson statistical 
nature of data it is necessary not to perform any pre-corrections (Qi et al 1998). Corrections for randoms, scatter and any other effects should be incorporated into the reconstruction procedure itself, rather than being applied as pre-corrections. At times, sophisticated rebinning strategies are employed to build sinograms into radial and angular sets, which changes the statistical distribution of the data, which may no longer be Poisson like (Kadrmas 2004). Furthermore, much attention must be paid in order that sinogram rebinning does not cause a loss in the potential accuracy of the reconstruction. Our approach does not involve sinograms in any way, thus preserving all the information obtained during the acquisition. Uncorrected data, binned into raw 3D-LOR histograms, should maintain Poisson statistics (Kadrmas 2004).

A serious disadvantage of the EM procedure is its slow convergence (Lewitt and Matej 2003). This is due to the fact that the image is updated only after a full iteration is finished, which implies that all the LORs have been projected and back projected at least once. In the ordered subset EM (OSEM) algorithm, proposed by Hudson and Larkin (1994), the image is updated more often, which has been shown to reduce the number of necessary iterations to achieve a convergence equivalent to that of EM.

According to the literature, EM methods have another important drawback: noisy images are obtained from over-iterated reconstructions, and this is usually attributed to either the fact that there is no stopping rule in this kind of iterative reconstruction or to the statistical (noisy) nature of the detection process and reconstruction method (Bettinardi et al 2002, Biemond et al 1990). In practice, however, an image of reasonable quality is obtained after a few iterations.

Several techniques have been proposed to address the issue of the noisy nature of the data: filtering the image either after completion of the reconstruction, during iterations or between them (Slijpen and Beekman 1999), removal of noise from the data using waveletbased methods (Mair et al 1996) or smoothing the image with Gaussian kernels (Sieves method) (Snyder et al 1987, Liow and Strother 1991).

Maximum a priori (MAP) algorithms are also widely used (Green 1990). MAP adds a priori information during the reconstruction process, the typical assumption being that due to the inherent finite resolution of the system the reconstructed image should not have abrupt edges. Thus, MAP methods apply a penalty function to those voxels which differ too much from their neighbours. Whether the maximum effective resolution achievable is limited, or even reduced, by the use of these methods is still an open issue. On the other hand, a proper choice of the reconstruction parameters, such as the number of iterations, the use of an adequate system response and a smart choice of subsetting, allows high quality images to be obtained by the EM procedure.

We implemented an OSEM algorithm that includes the possibility of MAP by means of a generalized one-step late MAP-OSEM algorithm, similar to the one described in Lewitt and Matej (2003) and Kadrmas (2004):

$$
x^{n, s+1}(j)=x^{n, s}(j)\left[\frac{\sum_{i \in \operatorname{Subset} \mathrm{S}} A(i, j) \frac{y(i)}{\left(R_{i}^{n}+S_{i}\right)}}{\sum_{i \in \operatorname{Subset} \mathrm{S}} A(i, j)(1+\operatorname{Penalty}(j, n))}\right]
$$

where the parameters and functions are defined as follows: $x(j)$ is the activity of voxel $j$ $(j=1$, maximum voxel number $V), x^{n, s}(j)$ is the expected value of voxel $j$ at iteration $n$ and subiteration $s, A(i, j)$ is the probability that a photon emitted from voxel $j$ is detected at LOR $i, y(i)$ is the projection from the object measured at LOR $i$ (experimental data), $S_{i}$ is the object scatter and random coincidences at $\operatorname{LOR} i$, Penalty $(j, n)$ is the penalty value at voxel $j$ and iteration $n, R_{i}^{n}$ is the projection estimated for the image reconstructed at iteration $n$ :

$$
R_{i}^{n}=\sum_{j=1}^{\text {Maximum voxel number }} A(i, j) x^{n}(j) .
$$


This MAP-OSEM algorithm can be considered as a generalization of the ML-EM. It incorporates a penalty MAP function which can be chosen in different ways (Stayman and Fessler 2001, Yu and Fessler 2002, Nuyts and Fessler 2003), and scatter and random counts' estimates that may require additional modelling of these processes. OSEM reconstruction without MAP regularization is obtained by setting the penalty function to zero. We note, however, that in this work we are mostly interested in the way we compress the SRM and not in the effect of MAP on the image quality, and thus all the reconstructed images we present are obtained with zero penalty.

With regard to the number of iterations and subsets, we have found that reconstruction with $25+25+50$ subsets exhibit a good compromise between resolution and reconstruction time. Thus, all the reconstructions presented in this work are obtained with three iterations of 25,25 and 50 subsets $(25+25+50)$, respectively.

The effect of scatter inside the object in 3D acquisitions of small animal has been studied recently (Yang et al 2004, Yongfeng Yang 2006). It has been measured as a non-negligible fraction of recorded events coming from scatter. An accurate modelling of scatter at the object during reconstruction may improve image quality. In this work, scatter inside the object has been estimated assuming an isotropic and homogeneous scatter distribution.

With regard to attenuation, as it is a relatively minor effect for small animal PET (Yao et al 2005) and our main aim is to test the adequacy of our compressed SRM and not the importance of attenuation, we have not included it in the reconstructed images shown.

\section{Simulation results}

\subsection{Test set}

To test our methodology, we first reconstructed data from different simulated phantoms: uniform cylinders and point sources in different axial and transaxial positions and simulated microresolution and Defrise phantoms. In order to study the linearity of the reconstruction method as well as the conservation of the number of counts and noise properties, a 'spiral phantom' was designed (figures 7-8). It is comprised of three cylinders (background) of $11.5 \mathrm{~mm}$ in diameter, each with two spirals inside: a hot one (activity four times greater than the background) and a cold one (activity four times lower than the background). The diameter of these spiral shape cylinders are 1.4, 2.2 and $2.6 \mathrm{~mm}$. Events were generated from these test sets using our own MC method, taking into account positron range and non-collinearity effects. Neither attenuation nor scatter within the object were included for these simulations. The response of the detector was also realistically simulated considering the main physical effects contributing to the spread of the energy among crystals due to scatter in the scintillators. For each study, 10 billion events were simulated and stored as projection data. We realized that the realistic model of detector response resulted in wider CHORs, which contained many more voxels than when more simplistic models of the system response are used. The images reconstructed from these simulations have a resolution of $175 \times 175 \times 62$ voxels. The size of the phantoms and the images were chosen to be the same as the FOV of the eXplore Vista-DR (GE), namely $65 \mathrm{~mm}$ in diameter. Thus, the voxel size is $0.38 \times 0.38 \times 0.78 \mathrm{~mm}^{3}$.

\subsection{Evaluation}

Initial tests were run to verify that the compressed SRM and the uncompressed SRM yielded images of similar quality and with no artefacts (see figure 5). We will comment in more detail on the effect of compression in the SRM in the reconstructed images in the next section.

In a second step, an estimate of the point spread function (PSF) was obtained by using a phantom consisting of an array of small sources located at different radial and axial positions 


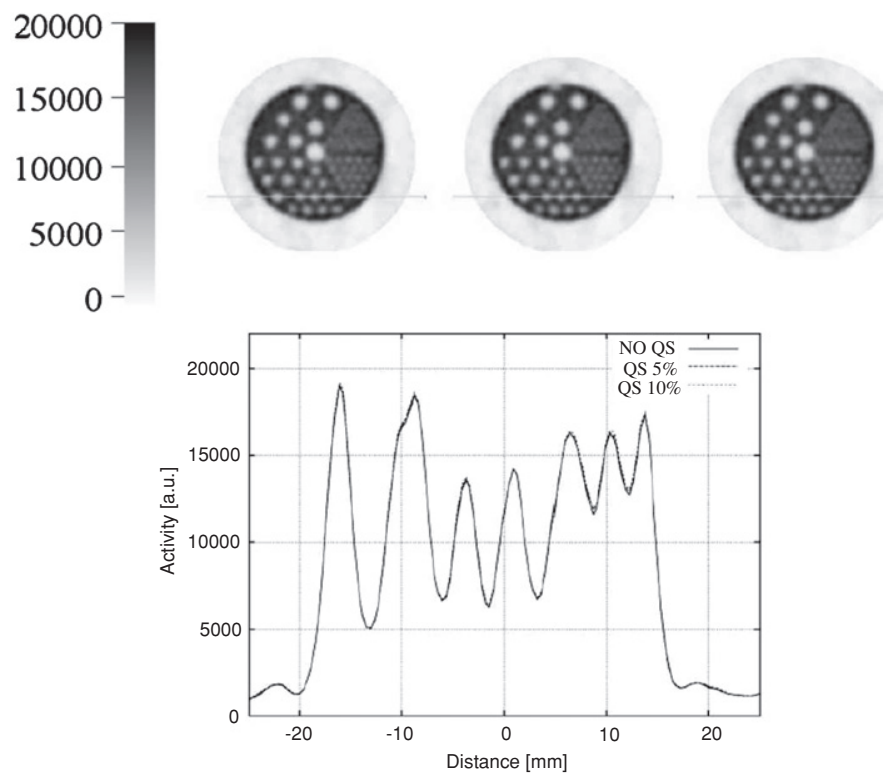

Figure 5. Reconstructions with different degree of quasi-symmetry assumptions. Transaxial slices of a $25+25+50$ 3D-OSEM reconstruction of a cold Derenzo phantom $\left(1 \mathrm{mCi}\right.$ of ${ }^{68} \mathrm{Ge}, 90 \mathrm{~min}$ acquisition time) are shown at the top panel. Left slice is obtained without quasi-symmetries. The centre one has been reconstructed using the quasi-symmetries explained in figures $2-4$. With this degree of quasi-symmetry, differences of less than 5\% inside superCHORs are found. The right slice has been obtained with a higher degree of quasi-symmetries (and compression), meaning up to $10 \%$ of difference of the profiles inside a superCHOR. At the bottom panel, the activity profile against the distance (in $\mathrm{mm}$ ) from the centre of the line drawn in the slices is shown. Small differences in the activity profile begin to be visible at the highest level of quasi-symmetry. Horizontal scale in $\mathrm{mm}$. Darker grey in the figure corresponds to larger activity values.

Table 2. Spatial resolution.

\begin{tabular}{lll}
\hline Measured & $R=0 \mathrm{~mm}$ & $R=25 \mathrm{~mm}$ \\
Radial & $0.7 \mathrm{~mm}$ & $0.9 \mathrm{~mm}$ \\
Tangential & $0.7 \mathrm{~mm}$ & $0.9 \mathrm{~mm}$ \\
Simulated (MC) & $R=0 \mathrm{~mm}$ & $R=25 \mathrm{~mm}$ \\
Radial & $0.6 \mathrm{~mm}$ & $0.8 \mathrm{~mm}$ \\
Tangential & $0.6 \mathrm{~mm}$ & $0.8 \mathrm{~mm}$ \\
\hline
\end{tabular}

and separated by $5 \mathrm{~mm}$. Resolution obtained from reconstructions of simulated projections as well as from real phantoms are shown in table 2, revealing that submillimetre resolution can be obtained from real projections. As shown in the figures and summarized in table 2, very uniform values of resolution (as measured by FWHM) throughout the FOV of $0.7 \mathrm{~mm}$ (at centre of the scanner) to $0.9 \mathrm{~mm}(2.5 \mathrm{~cm}$ off axis) were obtained.

These results of resolution can also be observed with the microresolution phantom reconstruction displayed in figure 6 , where the uniform resolution, almost constant along the radial direction, can be observed.

With regard to linearity, figures 7 and 8 show an spiral phantom and the reconstructed (OSEM) image after three iterations of $25+25+50$ subsets. Note the very linear response exhibited by the reconstructed image: the hot spiral to background and background to cold spiral activity ratios are preserved after reconstruction. 

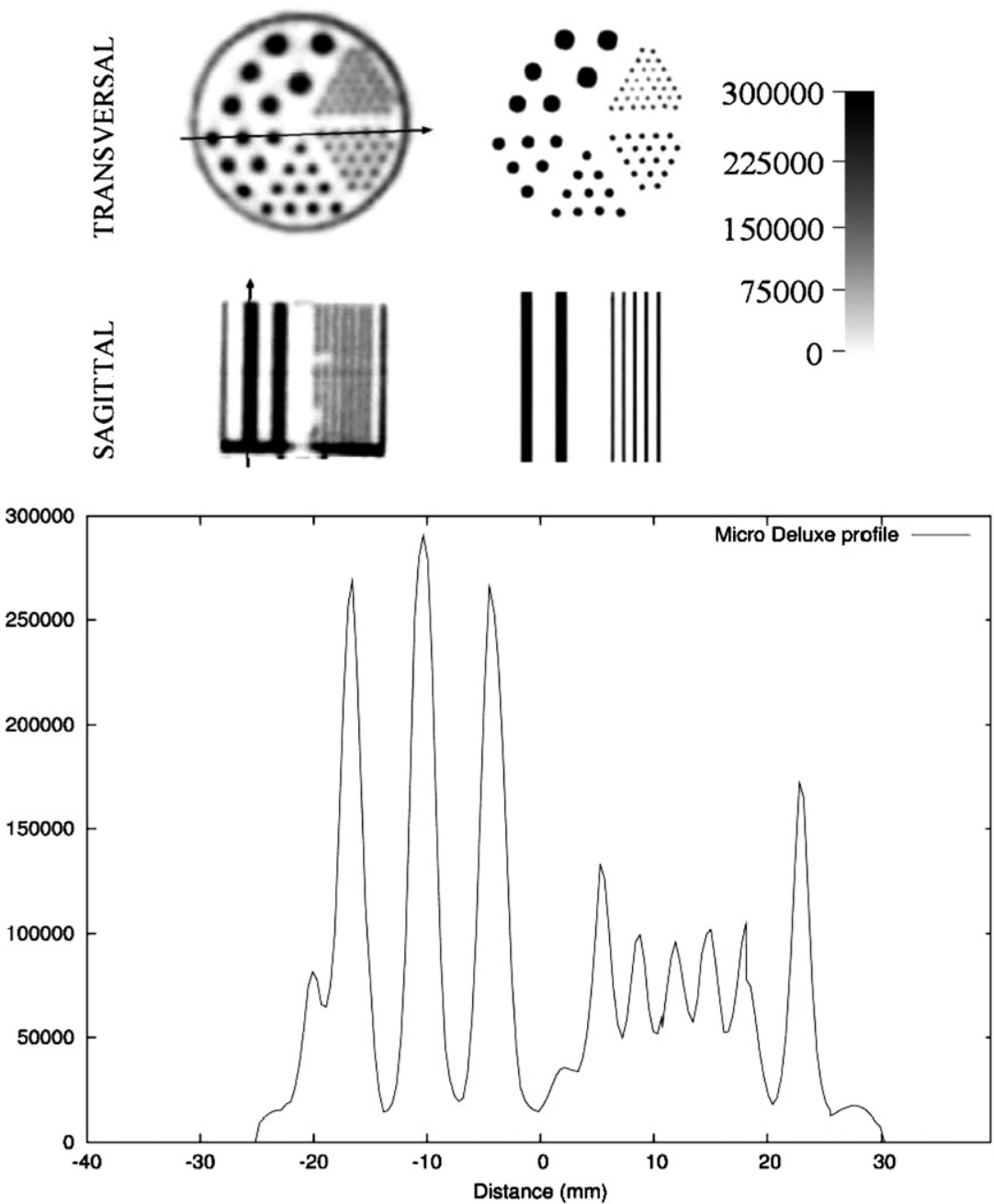

Figure 6. Microresolution phantom, Data Spectrum Co., Hillsborough, NC. Top: image reconstructed from real measured projections and from projections obtained after a simulation. Transversal and sagittal views. Rod diameters: $1.2,1.6,2.4,3.2,4.0$ and $4.8 \mathrm{~mm}$. Separation $=$ twice diameter. Bottom: transverse line profile of the measured microresolution phantom along the arrow indicated in the figure is shown.

\section{Evaluation of the effect of compression}

To study the effect of the quasi-symmetries we have implemented, we have chosen a $90 \mathrm{~min}$ acquisition of a cold Derenzo phantom of $1 \mathrm{mCi}$ activity of ${ }^{68} \mathrm{Ge}$. In figure 5 , we show a slice of the phantom reconstructed after three 3D-OSEM iterations of $25+25+50$ subsets where the SRM was dealt for in three ways. (a) Without making use of the quasi-symmetries. (b) With the quasi-symmetries explained in previous sections, and quasi-symmetry classes (superCHORs) built from profiles that typically differ by less than $5 \%$. This allows for a reduction in a factor of approximately 9 in the size of the SRM that needs to be stored. (c) 


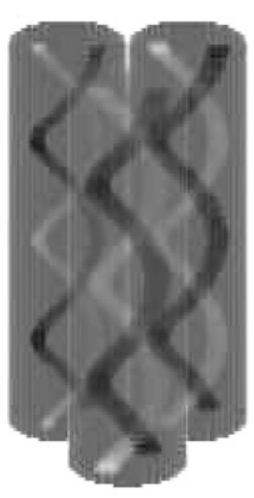

(a)

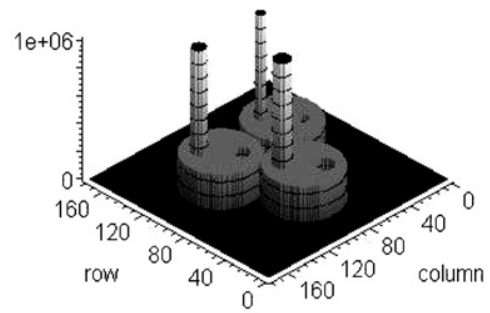

(b)

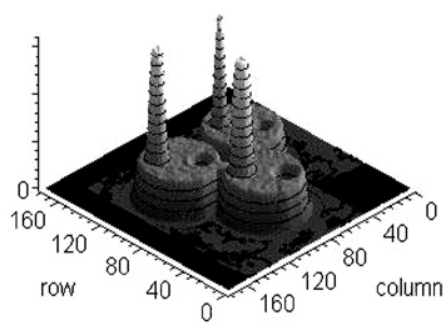

(c)

Figure 7. (a) Spiral phantom and (b) 3D representation of a transverse section of the original spiral phantom. Z-axis represents counts. (c) 3D representation of the OSEM-3D reconstructed image after three iterations $(25+25+50$ subsets). The three large cylinders are $11.5 \mathrm{~mm}$ in size, and there are two small ones inside each large cylinder, a hot one, with activity four times larger than the average on the large cylinder, and a cold one, with activity four times smaller than the average one. Small cylinders are 1.4, 2.2 and $2.6 \mathrm{~mm}$ in diameter.

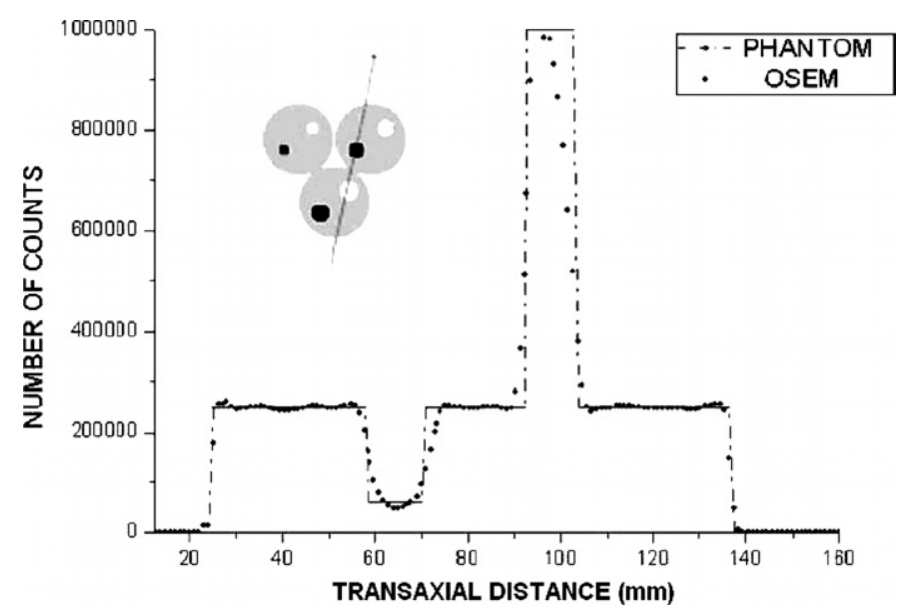

Figure 8. Profiles across the spiral phantom study showing the activity distribution: phantom (solid line), OSEM reconstruction (dots) $(25+25+50$ subsets). Voxel size is $0.38(X) \times 0.38(Y) \times$ $0.78(Z) \mathrm{mm}^{3}$.

With a larger degree of compression, which allows for a reduction factor in size of the SRM of approximately 25 , with superCHORs that represent profiles that differ approximately by less than $10 \%$. In the bottom part of the figure we show the activity profiles along the lines indicated in each slice of the upper part of the figure. While the activity profile of the reconstruction obtained without quasi-symmetry (solid line) and the one of the reconstruction obtained with moderate compression (labelled QS 5\%, medium dashed line) are hardly distinguishable, the 


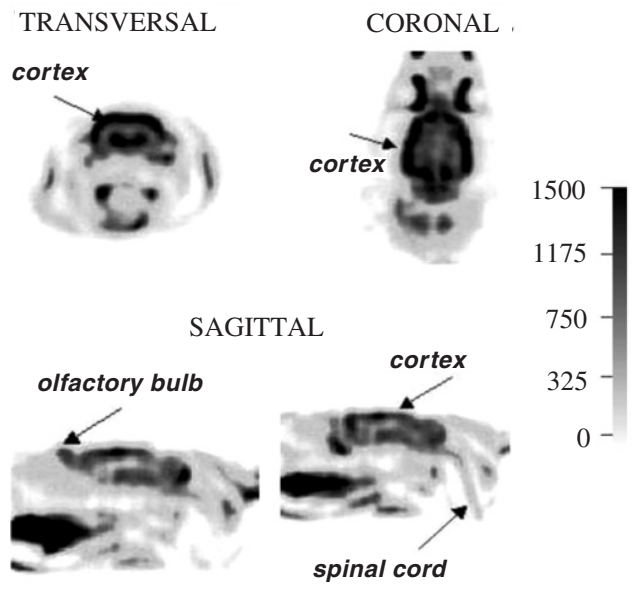

Figure 9. Single bed study of the head of a $185 \mathrm{~g}$ rat. $35 \mathrm{~min}$ intake of $1 \mathrm{mCi}$ of FDG and 60 min scan acquisition in an eXplore Vista (GE) drT PET scanner. 3D-OSEM with three iterations of $25+25+50$ subsets was employed.

reconstruction obtained with the most compressed SRM (labelled QS 10\%, short dashed line) begins to deviate slightly from the uncompressed result.

Apart from this figure where we have studied the effect of quasi-symmetries, in the remaining of this work we have employed the moderate amount of quasi-symmetries, which implies for the Vista drT scanner an SRM size of $150 \mathrm{MB}$.

\section{Results from small animal data}

Our reconstruction software was also tested on real mice data. ${ }^{18} \mathrm{~F}$ and FDG mice wholebody projections were acquired with a Vista (GE) drT PET scanner (Vaquero et al 2004). Figures 9 and 10 show the reconstructed images obtained using the 3D-OSEM algorithm with 3 iterations of $25+25+50$ subsets. The number of voxels is $175 \times 175 \times 62$ for the rat head depicted in figure 9 and $175 \times 175 \times 168$ for the whole body (three beds) mice of figure 10 . In all cases, the voxel size is $0.38 \times 0.38 \times 0.78 \mathrm{~mm}^{3}$. As indicated by the study from phantoms and simulated data, submillimetric details can be observed in the images of the mice and the rat head. In the rat head, cortex, spinal cord and olfactory bulb are easily identified. For the mice results, the fluorine image clearly shows small details such as ribs and spinal bones, and the small bones in the front legs. The FDG image shows the usual accumulation of activity at the mouse urinary bladder, but no artefacts are produced in its vicinity.

\section{Performance analysis}

\subsection{Optimization techniques during 3D-OSEM reconstruction}

Considering all the strategies described previously, we implemented an accelerated version of OSEM that can optionally incorporate a penalty function in the reconstruction process (MAP-OSEM). The number of subsets in each iteration can be chosen freely in between 1 and 100, not limited by system symmetries. Subsetting strategies require that all the subsets have CHORs evenly distributed along the FOV. In order to achieve this, we pick superCHORs in random order and assign them consecutively to each subset. As all the CHORS belonging to the a superCHOR lie within the same subset, we can take advantage of symmetries and quasi-symmetries to speed up decompression of the SRM, because every CHOR needs to be 


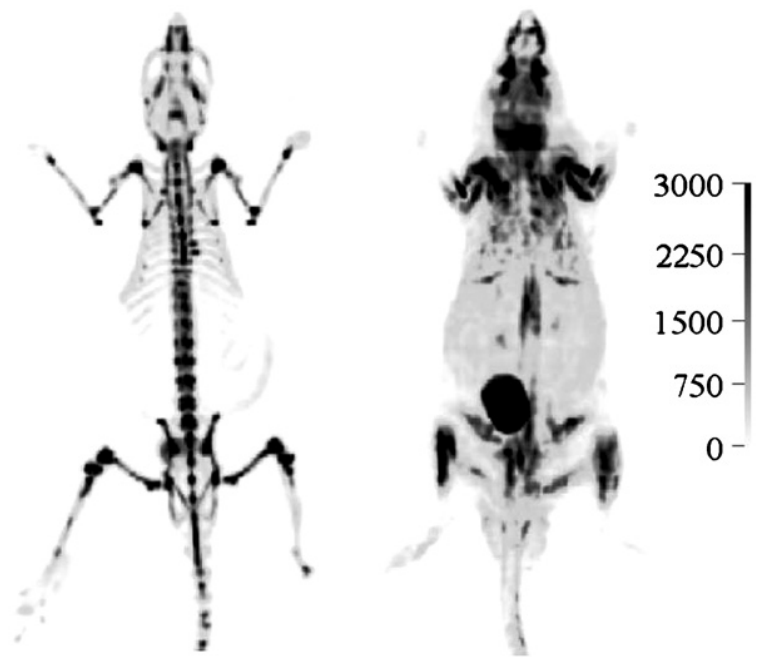

Figure 10. Reconstructed images of (a) ${ }^{18} \mathrm{~F}$ and (b) FDG mouse study acquired with an eXplore Vista (GE) drT PET scanner. A three-bed scan of a $25 \mathrm{~g}$ mouse, with 5 min scan acquisition per bed, four slices overlap between beds, $45 \mathrm{~min}$ uptake of $250 \mu \mathrm{Ci}$ of FDG (right) or ${ }^{18} \mathrm{~F}$ (left). 3D-OSEM with three iterations of $25+25+50$ subsets were employed in both cases.

decompressed once and only once during each iteration. Subsets are thus chosen so that they include all members of a quasi-symmetry class. From 1 to 100 subsets, there are plenty of choices to build the subsets that fulfil this requirement of including all the members of the classes comprised in a subset. We just chose symmetry classes in each subset at random. Beyond 100 subsets, however, problems arise because every subset will then include too few symmetry classes.

Always keeping in mind flexibility as a goal of design, the number of subsets as well as the number and size of voxels or the size of the FOV employed can be changed at any time during reconstruction, even before full iterations are completed. In this way, we can try different sizes of voxel, number of subsets and iterations and look for the best combination in terms of speed, quality of the reconstruction or both. For instance, during the first stages of reconstruction, when only the low frequencies (gross details) of the image are recovered, the use of a high number of voxels is a waste of computer power. The number of voxels employed to represent the image may be increased as the high frequency components of the reconstructed image begin to appear. This strategy has been described in detail in Raheja et al (1999) and it has been named multiresolution. We note that the images and execution times quoted in present work have been obtained without resorting to this feature.

With regard to reconstruction times, a full iteration of an acquisition covering the whole axial FOV (that is, an acquisition of 'one bed' or $175 \times 175 \times 62$ voxels) typically takes 30 min using $1 \mathrm{CPU}$ (Opteron 244, $1800 \mathrm{GHz}, 2 \mathrm{~GB}$ RAM). Thus $90 \mathrm{~min}$ are needed for the single-bed, three-iteration reconstructions shown in figure 9 of this work in a single-CPU computer. For larger animal like rats that span a length larger than the axial FOV of $4.8 \mathrm{~cm}$, the bed that supports the animal is displaced during acquisition and thus several scans (bed positions) are acquired consecutively in order to cover the whole body. More axial slices will be acquired and reconstruction time will be increased proportionally to the size of the axial FOV.

Reconstruction time scales approximately with the product of the number of LORs $\left(2.9 \times 10^{7}\right)$ and the number of voxels in a LOR (on average 6000 for the standard resolution 
employed of $175 \times 175 \times 62$ voxels). Without compression, a similar reconstruction needs to access above $3 \mathrm{~GB}$ worth of SRM elements from disk for every subset, which slows downs the reconstruction by a factor of $10-50$, depending on disk speed and network activity.

The reduction of the storage needs for the SRM allows us to keep it in RAM. The code is implemented in a way that no disk I/O is needed in order to forward and backward project. The SRM is read at once at the beginning of the execution and the image is written to disk only after a full iteration is finished. Except for the short initial and final periods of intense disk I/O, the common Unix tools measure a CPU use larger than 99\%, which indicates that the elapsed time during execution is mostly CPU bounded. Determining the performance of computer codes, however, is a very subtle and non-trivial task. A program can be CPU bounded yet it may be wasting CPU cycles doing nothing useful.

CPU manufacturers often quote peak performance of modern CPUs, referring to ideal situations where no cache misses occur, burst mode access to memory is possible, the CPU internal pipelines are fully used, etc. For instance, a peak performance of two flops per CPU cycle is quoted for AMD Opteron CPUs, which refers to a single multiply and add instruction performed in a clock cycle. Real life applications depart from the ideal conditions and thus peak CPU performance is hardly achieved during sustained execution of complex codes. In order to assess the performance of the code, FIRST was compiled with the 8.1 version of the Intel Fortran compiler. The Intel vtune performance analysis and profiling tool was employed to determine performance and number of flops required by each routine. We conclude that during reconstruction $50 \%$ of peak performance was obtained in sustained fashion. We also determined that when our compressed SRM that fits in RAM memory is employed, the decompression time measured was in the range of 10-30\% of total reconstruction time, depending on the number of voxels chosen.

\subsection{Parallel implementation}

Parallel computing on multiple processors is an attractive option in order to reduce computational time. The use of protocols like the message passing interface (MPI) enables clusters of networked industry-standard PCs (Beowulf clusters) to be relatively easy configured as a multiprocessor unit. Several parallel implementations of the OSEM algorithm have been presented in other works (Johnson and Sofer 1999a, 1999b, Chen 1998). We have decided to implement a parallel version of our Fast Iterative Reconstruction Software (FIRST) to run in Beowulf clusters of several CPUs in a master/slave configuration, characterized by the use of a master process and several (usually as many as the number of available CPUs) slave processes. The master distributes the job among the slaves and continuously balances the workload to achieve the best performance taking into account differences in individual speed or workload on each CPU. In spite of its name, the master process does not perform any intensive calculations, though. On startup, the master process reads from disk (once and for all) the SRM elements and sends them to the slave processes. Enough RAM memory to store the full compressed SRM must be available for each slave process. After startup, the master process decides which part of each subset (i.e., which actual superCHORs of such subset) is forward and backward projected by each slave process. Once all the slave processes have finished with their share of each subset, the master process updates the image, that is also stored in memory, and broadcasts the new image to all the slave processes. Upon completion of the reconstruction, the master process updates the image on disk. The slave processes are highly CPU intensive, as they are continuously performing the forward and backward projection operations. 
Table 3. First reconstruction elapsed time. Elapsed times for reconstruction of 50 subsets for one bed reconstructed with $175 \times 175 \times 62$ voxels are displayed on dedicated machines. The same code compiled with Intel Fortran compiler 8.1 and 32 bits libraries is employed in both systems. Fedora core $3.0 \times 86-1386$ operating system. At least $1 \mathrm{~GB}$ per CPU is available in all cases. The elapsed time in minutes is shown. In both cases, the clusters were configured with dual nodes connected via GB Ethernet cards and switches.

\begin{tabular}{llll}
\hline CPUs & Version & CPU class & Elapsed time (min) \\
\hline 1 & Non-parallel & AMD Opteron 244 1.8 GHz & 32.3 \\
1 & 1 master + 1 slave & id. & 35.2 \\
2 & 1 master + 2 slaves & id. & 18.2 \\
4 & 1 master + 4 slaves & id. & 9.4 \\
8 & 1 master + 8 slaves & id. & 5.0 \\
& & & \\
1 & Non-parallel & Intel Xeon EMT64 2.8 GHz & 43.6 \\
1 & 1 master + 1 slave & id. & 46.1 \\
2 & 1 master + 2 slaves & id. & 24.3 \\
4 & 1 master + 4 slaves & id. & 12.2 \\
\hline
\end{tabular}

The master process only takes part in the reconstruction whenever one of the slave processes finishes its share of the reconstruction task and claims for more or when all the CHORs of a subset have been visited by all the slave processes and then the image must be updated and broadcasted. Multitask capabilities of modern computers and operating systems make it possible to have as many slave processes as available CPUs, yet having an additional master process that will occupy a few cycles of one of the CPUs that is running one of the slave process. Balance of the workload among different CPUs is easily achieved as the slave processes that run faster (because they are executed by a less busy or faster CPU) will claim for their share of subsets more often than the ones that run slower. The only caution that must be taken is that the initial workload sent to each slave process is similar but not identical for all of them. In this way, we minimize the possibility that more than one slave process claims the attention of the master process at the same time. In practice, the tasks assigned to the slave process take from a few seconds to near a few tenths of seconds to be completed before requesting the action of the master process. The master process, on the other hand, can comply with the task required by the slave process in just a fraction of a second.

Overall, the implementation is simple and efficient. In table 3, we quote the elapsed time in minutes taken by the reconstruction of one iteration of 50 subsets at nominal number of voxels of $175 \times 175 \times 62$. We made tests in a one-CPU system comparing the parallel to the nonparallel versions. A master plus a slave process parallel reconstruction in a single CPU takes less than $10 \%$ longer than the nonparallel (only one process in one CPU) version of the code working in the same single-CPU system. The additional time is due to the overhead of sending the SRM from the master to the slave, as well as the elements of the image after updates, using the MPI interface. On the other hand, the parallel version of FIRST working over several CPUs reduces the elapsed reconstruction time by a factor nearly equal to the number of CPUs available. For relatively small clusters with up to eight CPUs, the results shown in table 3 indicate that the implementation with only one master process is rather efficient. When two or more CPUs are available, the master process uses less than $2 \%$ of the total computing time required for the reconstruction, according to the CPU use stated by the Unix common tools function $p s$.

If a number of CPUs larger than eight is to be used, benefits will be found by using more than one master process. In table 3, a summary of elapsed time for the same reconstruction over two different platforms using one master process is shown. 


\section{Discussion and conclusions}

We implemented FIRST, a fully 3D-OSEM or 3D-MAP-OSEM non-sinogram-based reconstruction algorithm, using a compressed SRM that contains the resolution recovery properties of EM. The full SRM can be stored in less than $150 \mathrm{MB}$ of storage. Reconstructed images are indistinguishable from the ones obtained without compression. The use of the compressed SRM allowed for a reconstruction with a more realistic response of the system. In this work, we used our own MC model of the scanner which incorporates physical effects such as positron range, non-collinearity and scatter in the scintillator material. Although it took several weeks, the SRM was computed only once. It was stored in compressed form so that the reconstruction program could keep it in dynamic memory.

Thanks to this, near peak performance of the algorithm is achieved with just a slight overhead (10-30\%) due to the decompression procedure. This resulted in short reconstruction times, even if the realistic SRM implies wider CHORs and thus more voxels are involved in every projection and back projection operation than when simplified SRM are used. The algorithm has been validated against simulations as well as real data. Acquisitions of phantoms and mice from a commercially available high-resolution PET scanner were reconstructed. A realistic SRM from our own MC model of the scanner with optimal resolution recovery was used. This fact, together with the intrinsic high resolution (small crystal pitch of $1.55 \mathrm{~mm}$ ) of the scanner, resulted in very high quality images with submillimetre resolution, as shown in figures 6,9 and 10. The reconstruction time needed by the algorithm enables real time operation in a small cluster (less than 10 min per bed and iteration in a four CPUs cluster) of industry-standard PCs. The results from real acquisitions in terms of resolution and linearity agree with what is expected from the simulated projections that use the same SRM as the reconstructions. This indicates that the SRM derived from our Monte Carlo simulations accurately reflects the response of the real scanner. Very uniform resolution and linearity is exhibited by the reconstructed images.

The flexibility, reduced reconstruction time, accuracy and resolution of the resulting images prove that the methodologies used to implement the FIRST reconstruction can be applied to real studies of high-resolution small animal PET scanners. The use of quasisymmetries to reduce (compress) the size of the SRM seems to be an adequate way of dealing with the problem of storing the huge SRM resulting from modern high-resolution PET scanners.

\section{Acknowledgments}

The authors thank Dr Martin G Pomper, James Fox and Jürgen Seidel from the Johns Hopkins University School of Medicine for providing access to the eXplore Vista datasets. Part of this work is funded by the IM3 network (Ministerio de Sanidad), with grants from the Ministerio de Educación y Ciencia, projects BFM2003-04147-C02-01 and TEC2004-07052-C02-01, Fondo de Investigaciones Sanitarias del Instituto de Salud Carlos III project PI052583 and Ministerio de Industria, Turismo y Comercio projects FIT-330101-2004-3 and CDTEAM (CENIT).

\section{References}

Bettinardi V, Pagani E, Gilardi M, Alenius S, Thielemans K, Teras M and Fazio F 2002 Implementation and evaluation of a $3 \mathrm{~d}$ one-step late reconstruction algorithm for $3 \mathrm{~d}$ positron emission tomography brain studied using median root prior Eur. J. Nucl. Med. 29 7-18

Biemond J, Lagendijk R and Mersereau R 1990 Iterative methods for image deblurring Proc. IEEE 78 856-83 
Browne J and De Pierro A R 1996 A row-action alternative to the EM algorithm for maximizing likelihoods in emission tomography IEEE Trans. Med. Imaging 15 687-99

Chen C M 1998 An efficient four-connected parallel system for PET image reconstruction Parallel Comput. 24 1499-522

Frese T, Rouze N C, Bouman C A, Sauer K and Hutchins G D 2003 Quantitative comparison of fbp, EM, and Bayesian reconstruction algorithms, including the impact of accurate system modeling, for the IndyPET scanner IEEE Trans. Med. Imaging 22 258-76

Green P J 1990 Bayesian reconstructions from emission tomography data using a modified EM algorithm IEEE Trans. Med. Imaging 9 84-93

Hudson H M and Larkin R S 1994 Accelerated image reconstruction using ordered subsets of projection data IEEE Trans. Med. Imaging 13 601-9

Johnson C A and Sofer A 1999a Frontiers'99. 7th Symp. on the Frontiers of Massively Parallel Computation pp $126-37$

Johnson C A and Sofer A 1999b Proc. 7th Symp. on the Frontiers of Massively Parallel Computation (Los Alamitos, CA: IEEE Computer Society Press) pp 126-37

Kadrmas D J 2004 LOR-OSEM: statistical PET reconstruction from raw line-of-response histograms Phys. Med. Biol. 49 4731-44

Kudrolli H, Worstell W and Zavarzin V 2002 Ss3d-fast fully 3d PET iterative reconstruction using stochastic sampling IEEE Trans. Nucl. Sci 49 124-30

Lee J, Vaquero J J, Barbosa F J, Seidel J and Green M V 2000 High performance phoswich detector module for small animal PET J. Nucl. Med. 41 19P

Lee K, Kinahan P E, Fessler J A, Miyaoka J S, Janes M and Lewellen T K 2004 Pragmatic fully 3d image reconstruction for the mices mouse imaging PET scanner Phys. Med. Biol. 49 4563-78

Lewitt R M and Matej S 2003 Overview of methods for image reconstruction from projections in emission computed tomography Proc. IEEE 91 1588-611

Liow J S and Strother S C 1991 Practical tradeoffs between noise, resolution and quantitation, and number of iterations for maximum likelihood reconstructions IEEE Trans. Med. Imaging 10 563-71

Mair B A, Carroll R B and Anderson J M M 1996 Filter banks and the EM algorithm Nucl. Sci. Symp., 1996 Conf. Rec., 1996 IEEE vol 3 pp 2-9

Michel C et al 2000 IEEE NSS-MIC Conf. Rec. pp 15/207-15/212

Nuyts J and Fessler J A 2003 A penalized-likelihood image reconstruction method for emission tomography, compared to postsmoothed maximum-likelihood with matched spatial resolution IEEE Trans. Med. Imaging 1042-52

Qi J, Leathy R M, Cherry S R, Chatzioannou A and Farquhar T H 1998 High-resolution 3d Bayesian image reconstruction using the micropet small-animal scanner Phys. Med. Biol. 43 1001-13

Rafecas M, Mosler B, Dietz M, Pgl M, Stamatakis A, McElroy M P and Ziegler S I 2004 Use of a Monte Carlo based probability matrix for 3d iterative reconstruction of MADPET-II data IEEE Trans. Nucl. Sci. 51 2597-605

Raheja A, Doniere T F and Dhawan A P 1999 Multiresolution expectation maximization reconstruction algorithm for positron emission tomography using wavelet processing IEEE Trans. Nucl. Sci. 46 594-602

Shepp L A and Vardi Y 1982 Maximum-likelihood reconstruction for emission tomography IEEE Trans. Med. Imaging 1 113-21

Slijpen E T P and Beekman R J 1999 Comparison of post-filtering and filtering between iterations for SPECT reconstruction IEEE Trans. Nucl. Sci. 46 233-8

Snyder D L, Miller M I, Thomas L J and Politte D G 1987 Noise and edge artifacts in maximum-likelihood reconstruction for emission tomography IEEE Trans. Med. Imaging 6 228-38

Stayman W and Fessler J A 2001 IEEE NSS Conf. Rec. vol 2 pp 1060-3

Vaquero J J, Pascau J, Molins A, Arco J M and Desco M 2004 Performance characteristics of the ARGUS-drT small animal PET scanner: preliminary results IEEE NSS-MIC Conf. (Rome, Italy, 2004) (Book of Abstracts) p 148

Yamaga T and Murayama H 2002-2003 DOI-PET image reconstruction with accurate system modeling that reduces redundancy of the imaging system Technical Report National Institute of Radiological Sciences

Yang Y, Tai Y C, Siegel S, Newport D F, Bai B, Leahy Q L R M and Cherry S R 2004 Optimization and performance evaluation of the microPET II scanner for in vivo small-animal imaging Phys. Med. Biol. 49 2527-45

Yao R, Seidel J, Liow J S and Green M V 2005 Attenuation correction for the NIH ATLAS small animal PET scanner IEEE Trans. Nucl. Sci. 52 664-8

Yongfeng Yang C S R 2006 Observations regarding scatter fraction and NEC measurements for small animal PET IEEE Trans. Nucl. Sci. 54 127-32

Yu D F and Fessler J A 2002 Edge-preserving tomographic reconstruction with nonlocal regularization image processing IEEE Trans. Med. Imaging 21 159-73 